\title{
Comprehensive Analysis of Expression, Prognosis and Immune Infiltrates for Kinesin Superfamily Members in Human Pancreatic Adenocarcinoma
}

\section{Jie Wang \\ Wuhan University \\ Jian Xu \\ Wuhan University \\ Wenjie Zhu \\ Wuhan University \\ Jianxin Jiang \\ Wuhan University}

Zehao Chen ( $\nabla$ czh11200095@163.com )

Wuhan University https://orcid.org/0000-0001-9440-0782

\section{Research}

Keywords: Kinesin superfamily, Pancreatic Adenocarcinoma, Expression, Prognostic value, Immune Infiltrates

Posted Date: June 16th, 2021

DOI: https://doi.org/10.21203/rs.3.rs-550619/v1

License: (c) (i) This work is licensed under a Creative Commons Attribution 4.0 International License. Read Full License 


\section{Abstract}

\section{Background}

Kinesin superfamily (KIFs) has a long-reported significant influence on the initiation, development, and progress of pancreatic adenocarcinoma (PAAD). However, the expression level of different KIFs in PAAD and its relationship with the prognosis and immune infiltration in patients with PAAD have not been fully elucidated.

\section{Methods}

Comprehensive bioinformatics analyses were done using data from UCSC XENA data hubs, TCGA database $\mathbb{Z}$ Oncomine databases, GEO datasets, GEPIA, GTEx, The human protein atlas, Kaplan Meier plotter, cBioPortal, STRING and KEGG database. Then, the relationship between KIFs expression and tumor immune infiltrates was studied by using the TIMER database.

\section{Results}

A total of 24 differentially expressed KIFs at transcriptional levels were identified between tumor tissues and normal tissues with 1 (KIF1A) downregulated and 23 (KIF2A, KIF2C, KIF3A, KIF3B, KIF3C, KIF4A, KIF5B, KIF7, KIF9, KIF10, KIF11, KIF13A, KIF13B, KIF15, KIF16B, KIF18B, KIF20A, KIF20B, KIF21B, KIF22, KIF23, KIF26B, KIFC1) overexpressed by GEPIA. The protein expression level of KIF3A, KIF3B, KIF4A, KIF5B, KIF7, KIF9, CENPE, KIF11, KIF13A, KIF13B, KIF15, KIF16B, KIF18B, KIF20A, KIF20B, KIF21B, KIF22, $\mathrm{KIF} 23, \mathrm{KIF} 26 \mathrm{~B}, \mathrm{KIFC} 1$ are higher in PAAD tissues than in the adjacent tissues, which is almost the same as the results of KIFs at transcriptional levels in PAAD. In addition, the expression levels of KIF1A, KIF1C, KIF3A, KIF5A, KIF5C, KIF6, KIF9, KIF13B, KIF19A, KIF21A, KIF24, KIF26B, KIFC1 are significantly correlated with the tumor stage of PAAD patients. As for prognosis value, the abnormal expression of KIF2C, KIF4A, KIF11, KIF15, KIF18B, KIF20A, KIF20B, KIF21B and KIF23 in PAAD patients is significantly related to the worse overall survival (OS) and relapse-free survival (RFS), and based on this, a 5-KIFs-based (KIF15, KIF20A, KIF20B, KIF21B, KIF23) risk score was generated by LASSO regression with a nomogram validated an accurate predictive efficacy. GO and KEGG enrichments revealed functions and pathways affected in PAAD. We also found that the expression of the 5-KIFs was significantly correlated with immune infiltrates, including B cells, $C D 8+T$ cells, $C D 4+T$ cells, neutrophils, macrophages, and dendritic cells.

\section{Conclusion}

Our study may provide new insights into the choice of prognostic biomarkers and immunotherapy targets in PAAD patients.

\section{Background}


Pancreatic adenocarcinoma (PAAD) and its variants account for $90 \%$ of all pancreatic carcinomas[1]. PAAD is a kind of extremely malignant tumor of the digestive system that is difficult to diagnose and treat[2]. Due to the difficulty of early diagnosis, high surgical mortality, poor treatment outcome, the 5-year survival rate of PAAD patients is only 5\% [3]. Although PAAD treatment has been significantly improved in recent decades, most patients are diagnosed with cancer only in the middle and late stages because the early symptoms of PAAD patients are not obvious, and there is a lack of biomarkers for early diagnosis. It is not effective for surgical resection and ultimately leads to high mortality in patients with PAAD[4]. Therefore, it is urgent to find effective biomarkers for early diagnosis and prognosis of PAAD and develop new strategies for targeted therapies for PAAD.

Kinesin superfamily proteins (KIFs) were first identified by Vale et al. in 1985[5]. KIFs are a group of proteins featured to be microtubule-based motors and functioned as intracellular transporters that directionally transport various cargos, including organelles, protein complexes and mRNAs, along microtubules in an adenosine triphosphate (ATP)-dependent way and played crucial roles in cellular morphogenesis and fundamental biology, like mitosis and meiosis [6-8]. There are $45 \mathrm{KIFs}$ discovered and identified in human, among which several family members were demonstrated varied functions in tumor pathobiology [9]. KIF11 was identified as a molecular target that shuttles between the proliferation and invasion of glioblastoma [10]. KIF20A peptide-based immunotherapy for cancer treatment was demonstrated availability and putative efficacy with promiscuous T-H-cell epitopes derived from KIF20A identified in solid tumor tissue and distinguished KIF20A-specific TH1-cell responses were found in patients with HNMT receiving immunotherapy[11]. Microarray data analyses revealed the highly transactivated status of KIF4A in non-small cell lung cancer and targeting KIF4A might hold a promise for the development of anticancer drugs and cancer vaccines as well as a prognostic biomarker in the clinic[12]. Many researches were done highlighting the importance of KIFs in various aspects of breast cancer [13]. Given the essential roles of KIFs reported in cancer, KIF-targeting cancer therapies were highly expected to be of great efficacy. It is worth noting that several KIF-inhibitors were invented and tested in clinical trials. Ispinesib, a KIF11-targeted inhibitor, was the first KIF-inhibitor that was evaluated both safety and efficacy in breast cancer in phase I clinical study[14]. Other KIF-targeted drugs further tested in various cancers by clinical trials including KIF11 inhibitors (litronesib[15, 16], filanesib[17-19], SB743921[20], AZD4877[21]), KIF5C inhibitors (Lidocaine and Tetracaine[22]) and KIFC1 inhibitors (AZ82 and SR31527[23, 24]). However, despite numerous researches done, the prognostic and therapeutic value of all KIFs remains uncorroborated. Considering the intricate functions of KIFs in mitosis, singling out any particular KIFs may not be an efficient way to fulfill the therapeutic capacity of KIFs. At the same time, common regulatory network of all KIFs is little known, which may give new insight into the limited therapeutic efficacy shown in clinical trials and provide putative drug target by mutually regulating KIFs in cancer.

\section{Results}

\section{Expression profile of KIFs in PAAD}


The TCGA expression profile of all KIFs was shown in the heatmap by a dataset from UCSC XENA data hubs (Fig. 1). Distinct expressions profiles were seen between normal and tumor tissue. Clustering analysis found most KIFs overexpressed in tumor tissue. Then, comparisons were done between TCGA tumor samples and matched TCGA and GTEx normal samples in GEPIA dataset, a total of 24 differentially expressed KIFs were found. Among which, only 1 KIFs (KIF1A) showed decreased expression in tumor samples while $23 \mathrm{KIFs}$ (KIF2A, KIF2C, KIF3A, KIF3B, KIF3C, KIF4A, KIF5B, KIF7, KIF9, KIF10, KIF11, KIF13A, KIF13B, KIF15, KIF16B, KIF18B, KIF20A, KIF20B, KIF21B, KIF22, KIF23, KIF26B, KIFC1) significantly overexpressed in PAAD (Fig. 2,3). Then we study the expression profile of KIFs in PAAD tissues and normal tissues in Oncomine database (Table 1), which is almost correspond with the results in GEPIA dataset.

Figure 2 Expression boxplot of each kinesin superfamily members (KIF1A, KIF1B, KIF1C, KIF2A, KIF2B, KIF2C, KIF3A, KIF3B, KIF3C, KIF4A, KIF4B, KIF5A, KIF5B, KIF5C, KIF6, KIF7, KIF11, KIF9, KIF10, CENPE, KIF12, KIF13A, KIF13B, KIF14, KIF15, STARD9, KIF16B, KIF17, KIF18A, KIF18B, KIF19A, KIF20A, KIF20B, KIF21A, KIF21B, KIF22, KIF23, KIF24, KIF25, KIF26A, KIF26B, KIF27, KIFC1, KIFC2, KIFC3) using both TCGA-PAAD and matched GTEx-normal data (Tumor = 179; Normal $=171)$; $\mathrm{P}<0.05$; $*{ }^{*} \mathrm{P}<0.01$; $* \star * \mathrm{P}<$ 0.001

Table 1 Expression profile of KIFs in PAAD and normal tissues (Oncomine database). 


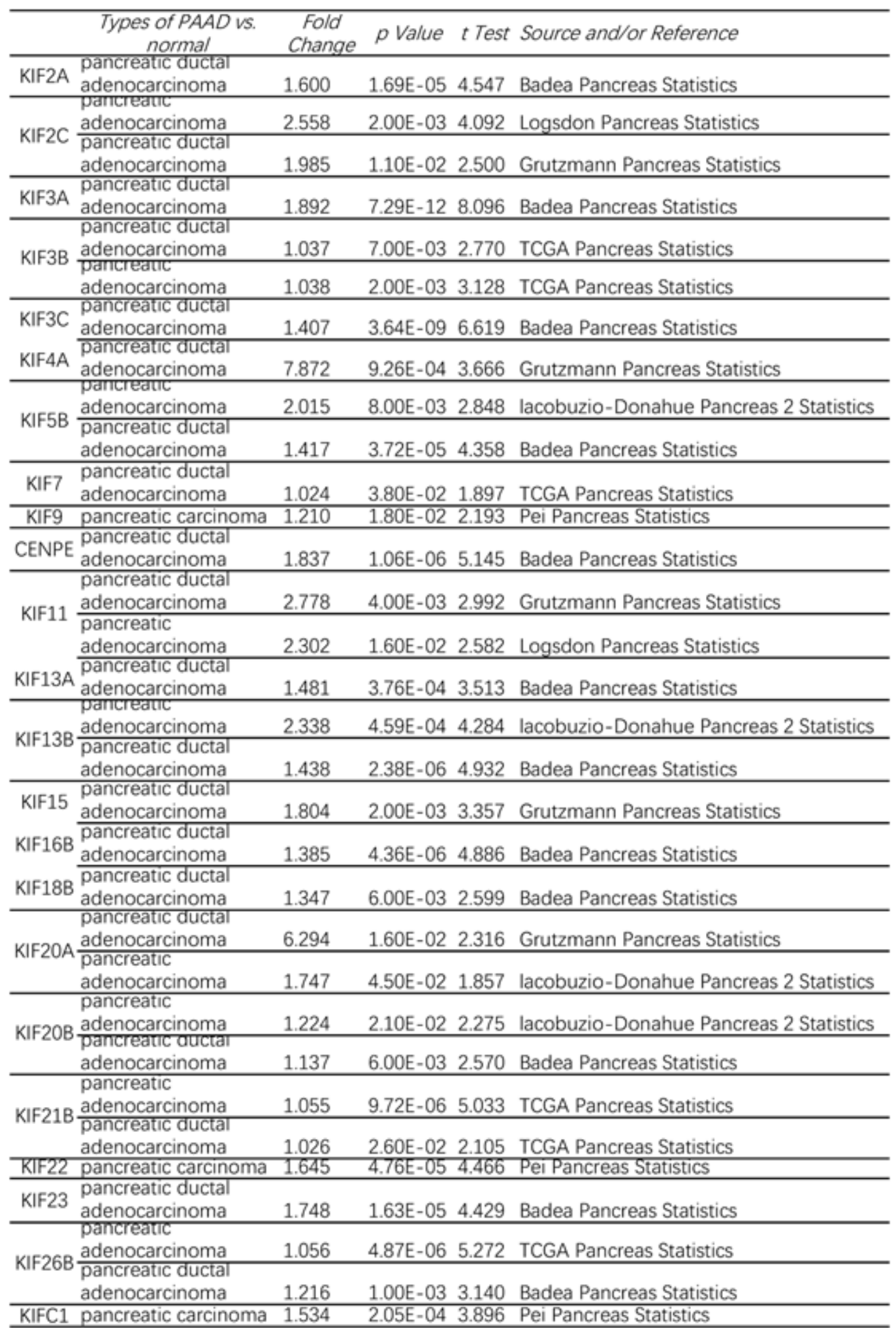

\section{Relationship Between the mRNA expression levels of KIFs and the tumor stage of PAAD Patients}

We also studied the relationship between mRNA expression levels of KIFs and tumor stage in PAAD patients by using the GEPIA dataset. In comparison of 178 PAAD patient samples and 171 normal samples, it showed that the expression levels of KIF1A, KIF1C, KIF3A, KIF5A, KIF5C, KIF6, KIF9, KIF13B, 
KIF19A, KIF21A, KIF24, KIF26B, KIFC1 are significantly correlated with the tumor stage of PAAD patients (Fig. 4), while the expression levels of the other KIFs are not correlated with the tumor stage of PAAD patients.

Figure 4 Correlation between KIFs Expression and Tumor Stage in PAAD Patients (GEPIA)

\section{The Protein Expression Level Of Kifs In Paad}

In The human protein atlas we found that the protein levels of KIF3A, KIF3B, KIF4A, KIF5B, KIF7, KIF9, CENPE, KIF11, KIF13A, KIF13B, KIF15, KIF16B, KIF18B, KIF20A, KIF20B, KIF21B, KIF22, KIF23, KIF26B, $\mathrm{KIFC1}$ are higher in PAAD tissues than in the adjacent tissues (Fig. 5), which is almost the same as the transcriptional levels between TCGA tumor samples and matched TCGA and GTEx normal samples.

Figure 5 The Expression of KIFs in PAAD (HPA)

\section{The Prognostic Value Of Kifs In Paad Patients}

To evaluate the value of KIFs at different transcription levels in the progression of PAAD, we evaluated the correlation between KIFs at different transcription levels and clinical outcome using Kaplan-Meier plotter analysis. In all 24 differentially expressed KIFs, after compared 89 high expression level patients with 89 low expression level patients, PAAD patients with high transcription levels of KIF21B were significantly associated with longer RFS and OS, in contrast, PAAD patients with high transcription levels of KIF2C, KIF4A, KIF11, KIF15, KIF18B, KIF20A, KIF20B, KIF23 were significantly related with worser OS and RFS (Fig. 6,7).

Figure 6 The prognostic value of different expressed KIFs in PAAD patients in the RFS curve (KaplanMeier plotter).

\section{Construction Of 5-kifs-based Risk Score}

Given the expression profile and survival analyses of all KIFs, 9 abnormal expression KIFs (KIF2C, KIF4A, KIF11, KIF15, KIF18B, KIF20A, KIF20B, KIF23, KIF21B) that demonstrated significant prognostic value in PAAD were enrolled for LASSO regression to construct a KIFs-based risk score for prediction of OS in PAAD. According to the cvfit plot, the minimal of lambda value was seen in 5, indicating a 5-KIFs-based risk score model the best for both accuracy and simplicity (Fig. 8a). Therefore, only KIF15, KIF20A, KIF20B, KIF21BA, KIF23 were included (Fig. 8b) and a 5-KIFs-based risk score was generated as below:

Rs $=0.12796255 \operatorname{Exp}($ KIF20A)-0.48974996 $\operatorname{Exp}($ KIF15) $+0.49730528 \operatorname{Exp}($ KIF20B $)+0.21624084 \operatorname{Exp}$ (KIF21B)- 0.07107506Exp (KIF23)

Rs: risk score; $\operatorname{Exp}(X)$ : the expression level of gene $X$ 
Validations of the 5-KIFs-based risk score done by Kaplan-Meier plots demonstrated good prognostic value in predicting OS of PAAD, with higher risk score significantly correlated with worse outcomes in both TCGA-PAAD data $(P=0.00076)$ and dataset (GSE62452) from GEO $(P=0.031)$ (Fig. 8c).

\section{Multivariate Analysis And Nomogram}

To evaluate the prognostic value of the risk score, we performed multivariate analyses on all demographic and clinical parameters (age, family history of cancer, history of chronic pancreatitis, history of diabetes, maximum tumor dimension, tumor grade, number of positive lymphnodes, radiation therapy, TNM stage and risk score) in TCGA dataset. Then we selected several indicators (age, number of positive lymphnodes, risk score) with significant differences for next step. A nomogram predicting 1-year and 3year OS of PAAD patients was constructed combining age, number of positive lymphnodes and LASSO regression generated 5-KIFs risk score using R package "rms" [46, 47]. Assessment of predictive accuracy was done by calibration plot[48].

\section{Genetic Alteration, Enrichment of co-expression genes, Strings, GO and KEGG Analysis}

The genetic alteration was analyzed by cBioPortal (www.cbioportal.org)[49]. The genomic map includes data for putative copy-number alterations (CNA) from GISTIC, mRNA expression z-scores and mutations. Co-expression genes correlated with KIFs were enriched using GEPIA by TCGA-PAAD data. STRINGS (www.string-db.org) is an online analysis tool that collects, scores and integrates all publicly available sources of protein-protein interaction (PPI) data and supplements it with calculations and predictions[50]. In this study, we performed a PPI network analysis on co-expression genes of KIFs to explore their interactions. The intersection of co-expressed genes was put into GO, KEGG and transcription factors enrichment using R package "ClusterProfiler"[51]. Enrichment results were represented as bubble plot using R package "ggplot"[52].

The functions of the 5-KIFs and the genes significantly associated with alterations were predicted by analyzing gene ontology (GO) and Kyoto Encyclopedia of Genes and Genomes (KEGG) in the Database for Annotation, Visualization and Integrated Discovery (DAVID) (https://david.ncifcrf.gov/). GO enrichment analysis predicted the functional roles of target host genes based on three aspects, including biological processes, cellular components, and molecular functions. We found that G0:0140014 (Mitotic Nuclear Division), GO:0048285 (Organelle Fission), GO: 0000070 (Mitotic Sister Chromatid Segregation), and GO: 0000280 (Nuclear Division) were significantly regulated by the KIFs alterations in PAAD (Figure $11 \mathrm{~A}, \mathrm{~B}, \mathrm{C})$.

KEGG analysis can define the pathways related to the functions of KIFs alterations and the frequently altered neighbor genes; 5 pathways significantly related to the functions of KIFs alterations in PAAD were found through KEGG analysis (Figure 11D). Among these pathways, hsa04110: Cell cycle, hsa04114: Oocyte Meiosis, hsa04914: Progesterone-Mediated Oocyte Maturation, has04115: P53 Signaling 
Pathway and has04218: Cellular Senescence were involved in the tumorigenesis and pathogenesis of PAAD.

\section{The Relationship Between KIFs Expression Levels and Immune Infiltration Levels in PAAD}

TIMER online analysis tool is used to evaluate the relationship between the transcription level of KIFs and the level of immune infiltration in PAAD. It was found that KIFs are involved in immune cell infiltration, which affect the clinical outcome of PAAD patients. The analysis results are shown in Figure 12. These studies indicate that the level of KIFs expression is associated to the level of immune infiltration in PAAD. Among the 5-KIFs, KIF15 expression was positively correlated with infiltration of B cells. KIF20A expression was positively correlated with the infiltration of B cells, neutrophil and dendritic cells while it was negatively correlated with infiltration of CD4+ T cells. KIF20B expression was positively correlated with the infiltration of CD8+ T cells, B cells, macrophage, neutrophil and dendritic cells while it was negatively correlated with infiltration of CD4+ T cells. KIF21B expression was positively correlated with infiltration of CD4+ T cells, CD8+ T cells, B cells, neutrophil and dendritic cells. KIF23 expression was negatively correlated with infiltration of CD4+ T cells, while it was positively correlated with infiltration of $B$ cells, neutrophil and dendritic cells. These studies indicate that the level of KIFs expression is associated to the level of immune infiltration in PAAD.

Based on above results, we selected immune $B$ cell to construct a Cox regression with KIF15, KIF20A, KIF20B, KIF21BA and KIF23. Therefore, TIMER online tool was used to proofread B cell, the 5-KIFs covariate factors and automatically output Cox regression results. These studies indicate that the KIF15 $(P=0.027), \operatorname{KIF} 21 B(P=0.039)$ and KIF23 $(P=0.024)$ was significantly correlated with the clinical outcomes of patients with PAAD (Table 2).

Table 2 The cox proportional hazard model of the 5-KIFs and B immune cells in PAAD (TIMER).

\begin{tabular}{ccccccc}
\hline & coef & HR & 95\%Cl_I & $95 \%$ Cl_u & P-value & sig \\
\hline B cell & -0.007 & 0.993 & 0.091 & 10.820 & 0.995 & \\
KIF15 & -0.642 & 0.526 & 0.299 & 0.928 & 0.027 & $*$ \\
KIF21B & -0.413 & 0.661 & 0.447 & 0.979 & 0.039 & $*$ \\
KIF20A & 0.216 & 1.241 & 0.687 & 2.243 & 0.474 & \\
KIF20B & 0.289 & 1.335 & 0.738 & 2.416 & 0.339 & \\
KIF23 & 0.714 & 2.043 & 1.098 & 3.800 & 0.024 & $*$ \\
\hline
\end{tabular}

\section{Discussion}

The KIF proteins participate in multiple normal cellular biological activities including mitosis and intracellular transport of vesicles and organelles[6]. Increasing evidence indicates kinesin proteins play critical roles in the genesis and development of human cancers. Some kinesin proteins are associated with malignancy as well as drug resistance of solid tumor. Thus, targeting KIF therapy seems to be a 
promising anticancer strategy. Therefore, comprehensive bioinformatics analyses were done in our study using data from multi-dataset to explore the expression, prognosis as well as Immune infiltrates of kinesin superfamily in PAAD patients. A total of 24 differentially expressed KIFs were identified between tumor tissue and normal tissue with 1(KIF1A) downregulated and 23 IKIF2A, KIF2C, KIF3A, KIF3B, KIF3C, KIF4A, KIF5B, KIF7, KIF9, KIF10, KIF11, KIF13A, KIF13B, KIF15, KIF16B, KIF18B, KIF20A, KIF20B, KIF21B, KIF22, KIF23, KIF26B) overexpressed. Survival analyses revealed 9 abnormal expressed KIFs (KIF2C, KIF4A, KIF11, KIF15, KIF18B, KIF20A, KIF20B, KIF21B, KIF23) significantly correlated with worse OS and RFS of PAAD patients, indicating efficient biomarkers for predicting the prognosis of PAAD. Further analyses were done with a 5-KIFs-based risk score generated by LASSO regression, a nomogram was constructed with elements selected by multivariate survival analysis and an accurate predictive efficacy was validated by another dataset.

KIF15 plays an important role in several kind of tumors. As reported, KIF15 is required for maintenance of spindle bipolarity and is the breast cancer tumor antigen[25]. In our group's early study, we reported for the first time that KIF15 promotes pancreatic cancer cell proliferation by promoting G1/S phase transition via regulating the MEK-ERK signaling pathway[26].

KIF20A has been documented to accumulate in the midzone of the spindle during anaphase and to the cleavage furrow and midbody during telophase[27]. It has been reported that KIF20A is overexpressed in human pancreatic ductal adenocarcinoma (PDAC) based on cDNA microarray analyses and that KIF20A and DLG5, which collaborate in the cytoplasm but not in the midzone of the spindle, are likely to be involved in pancreatic carcinogenesis[28]. KIF20A functions as part of the intracellular trafficking machinery for DLG5, transporting it to membrane sites in PDAC cells in which DLG5 can interact with other proteins including $\beta$-catenin[28]. Therefore, it is possible that the intracellular trafficking function of KIF20A is highly important for pancreatic carcinogenesis.

KIF20B is strongly overexpressed in bladder cancer tissues and downregulation of endogenous KIF20B leads to cytokinesis defect[29]. It's also reported that in multiple cancer cells, knockdown of KIF20B not only dramatically inhibits tumor cell growth, but also causes mitotic arrest, senescence and postmitotic apoptosis[30].

KIF21B is not only a classic kinesin protein but also a regulator of microtubule dynamics[31]. In vitro reconstitution studies show that KIF21B increases the microtubule growth rate and catastrophe frequency. Moreover, the purified protein surprisingly associates primarily with depolymerizing microtubule and microtubule ends[32]. A study published in 2017 confirmed that KIF21B is a potential microtubule-pausing factor[33]. Microtubules and kinesins play roles in intercellular signal transduction, transport, malignant tumorigenesis, and tumor progression, invasion, and metastasis[34, 35].

KIF23 is essential for the completion of cytokinesis in midbody formation and for recruitment to the spindle midzone/midbody by a chromosomal passenger protein[36]. In a study, it discovered the high expression of KIF23 was associated with poor prognosis by immunohistochemical analysis of tumor sections and clinical data from patients with PDAC[37]. They also found that knockdown of KIF23 
decreased proliferation in PANC-1 and BxPC-3 PDAC cells, and suppression of KIF23 in PANC-1 cells inhibited tumor growth in nude mice[37].

Furthermore, the mutation rate (6\%) of the 5-KIFs in PAAD is low, which indicates the expression of the 5KIFs is stable relatively. Then we analyzed the function of the 5-KIFs and its neighboring proteins by GO enrichment analysis and KEGG pathway enrichment. Studies have found that the functions of these proteins are mainly related to the Cell cycle, Oocyte Meiosis, Progesterone - Mediated Oocyte Maturation, P53 Signaling Pathway and Cellular Senescence, and some of these pathways are significantly associated with the occurrence and development of PAAD. These data indicate that differentially expressed KIFs in PAAD are potential targets for drug therapy.

Another important result of this study is that the transcription levels of the 5-KIFs is closely correlated with various levels of immune infiltration in PAAD. The correlation between the expression of the 5-KIFs and the marker genes of PAAD immune cells indicates that KIFs may be involved in the regulation of PAAD tumor immunity.

In conclusion, KIFs not only can be used as a prognostic indicator of patients with PAAD, but also reflect their immune status. These findings may help better study the molecular basis of PAAD and may help create more appropriate prognostic tools for PAAD and facilitate the development of new immunotherapeutic. However, more experimental studies are needed to confirm our results, thereby promoting the clinical application of KIFs as a prognostic indicator or immunotherapy target in PAAD.

\section{Conclusion}

Our research comprehensively illustrates the expression of KIFs in PAAD. We also built a risk score model of PAAD patients based on the TCGA database and it can still accurately predict the prognosis of PAAD patients in independent GEO data set. And we found that KIFs are involved in immune cell infiltration. The cox regression of several KIFs and immune cell can also affect the clinical outcome of PAAD patients. In summary, our study may provide new choices of prognostic biomarkers and immunotherapy targets in PAAD patients.

\section{Materials And Methods}

\section{TCGA Data and UCSC XENA data hubs Analysis}

KIFs RNA sequencing expression data of PAAD from the cancer genome atlas (TCGA, http://cancergenome.nih.gov/) were downloaded using UCSC XENA data hubs (https://tcga.xenahubs.net), the heatmap was drawn using R(4.0.2) package "pheatmap" with clinical parameters from TCGA-PAAD dataset[38].

\section{Gepia Dataset And Oncomine Databases Analysis}


Gene Expression Profiling Interactive Analysis (GEPIA, www.gepia.cancer-pku.cn) is a newly developed interactive web server that analyzes RNA sequencing expression data from 9,736 tumors and 8,587 normal samples from the Cancer Genome Atlas (TCGA) and Genotypic Tissue Expression (GTEx) projects using standard processing pipelines (www.gepia.cancer-pku.cn/). GEPIA can provide customizable functions, including differential expression analysis, patient survival analysis, cancer type staging, cancer pathological staging, correlation analysis, similar gene detection and dimensionality reduction analysis[39]. Oncomine gene expression array dataset (www.oncomine.org) is a publicly accessible online cancer microarray database for analyzing the KIFs transcription levels in different cancers[40]. Student's $t$ test was used to compare the transcription levels of KIFs in clinical cancer specimens with that in normal controls. The cut-off of P-value was defined as 0.05 .

\section{The Human Protein Atlas Analysis}

The Human Protein Atlas(HPA, https://www.proteinatlas.org/) is a Swedish-based program initiated in 2003 with the aim to map all the human proteins in cells, tissues and organs using an integration of various omics technologies, including antibody-based imaging, mass spectrometry-based proteomics, transcriptomics and systems biology[41]. We can find the expression levels of KIFs in PAAD tissues and its adjacent tissues.

\section{The Kaplan-meier Plotter Analysis}

The Kaplan-Meier plotter is an online database (www.kmplot.com) that contains gene expression data and survival information for clinical cancer patients[42, 43]. We used this online tool to assess the prognostic value of KIFs mRNA expression in PAAD patients and analyzed the OS and RFS of patients with PAAD. The database divides patient samples into high expression groups and low expression groups according to the median values of KIFs mRNA expression and validates them by Kaplan-Meier survival curve. Information on number of patients, median values of mRNA expression, $95 \%$ confidence interval $(\mathrm{Cl})$, hazard ratio (HR), and P-value can be found on the Kaplan-Meier plotter web page. P-value $<0.05$ was considered as statistically significant.

\section{Lasso Regression}

LASSO Cox regression is a widely used method for high-dimensional predictors selection[44]. In this study, TCGA-PAAD data were used to construct a risk score of KIFs for the prediction of OS. R package "glmnet" was used to execute LASSO Cox regression model analysis[45]. Cvfit plot was drawn, and the minimum lambda value was used as cutoff. The predictive model was validated in TCGA-PAAD and GEO datasets using KM plotter as previously described. 


\section{Timer Analysis}

TIMER (www.cistrome.shinyapps.io) is a convenient and accurate online analysis tool that can infer the abundance of tumor-infiltrating immune cells from gene expression profiles and evaluate their clinical impact[53]. In this study, we used TIMER to assess the correlation between KIFs expression levels and immune cell infiltration. And we also assess the correlation between clinical outcomes and immune cell infiltration and KIFs expression.

\section{Statistics}

For all the analyses done above, a P-value $<0.05$ was considered statistically significant except for specifically mentioned.

\section{Declarations}

Founding: This study was funded by the National Natural Science Foundation of China (81871965).

Consent for publication: Not applicable.

Author contributions $\ Z$ Zehao Chen conceived the project and wrote the manuscript. Jie Wang, Jian Xu and Wenjie Zhu participated in data analysis. Jie Wang, Jian Xu and Wenjie Zhu participated in discussion and language editing. Jianxin Jiang reviewed the manuscript. All authors contributed to the article and approved the submitted version.

Competing interests: The research was conducted in the absence of any commercial or financial relationships that could be construed as a potential conflict of interest. The authors declare that they have no competing interests.

Availability of data and materials: The datasets generated and/or analyzed during the current study are available in the UCSC XENA repository(https://tcga.xenahubs.net), Cancer Genome Atlas (TCGA, http://can-cergenome.nih.gov/), the GTEx projects and Gene Expression Omnibus (GEO, https://www.ncbi.nlm.nih.gov/geo/).

Ethics approval and consent to participate: Not applicable.

Acknowledgments: This study was funded by the National Natural Science Foundation of China (81871965).

\section{References}

1. Feldmann G, Beaty R, Hruban RH, Maitra A. Molecular genetics of pancreatic intraepithelial neoplasia. J Hepato-biliary-Pancreat Surg. 2007;14(3):224-32. 
2. Siegel RL, Miller KD, Jemal A. Cancer statistics, 2018. CA Cancer J Clin. 2018;68(1):7-30.

3. Ilic M, Ilic I. Epidemiology of pancreatic cancer. World J Gastroenterol. 2016;22(44):9694-705.

4. Samuel N, Hudson TJ. The molecular and cellular heterogeneity of pancreatic ductal adenocarcinoma. Nature reviews Gastroenterology hepatology. 2011;9(2):77-87.

5. Vale RD, Reese TS, Sheetz MP. Identification of a novel force-generating protein, kinesin, involved in microtubule-based motility. Cell. 1985;42(1):39-50.

6. Miki H, Okada Y, Hirokawa N. Analysis of the kinesin superfamily: insights into structure and function. Trends in cell biology. 2005;15(9):467-76.

7. Hirokawa N, Noda Y, Tanaka Y, Niwa S. Kinesin superfamily motor proteins and intracellular transport. Nature reviews Molecular cell biology. 2009;10(10):682-96.

8. Hirokawa N, Noda Y. Intracellular transport and kinesin superfamily proteins, KIFs: structure, function, and dynamics. Physiological reviews. 2008;88(3):1089-118.

9. Miki H, Setou M, Kaneshiro K, Hirokawa N. All kinesin superfamily protein, KIF, genes in mouse and human. Proc Natl Acad Sci USA. 2001;98(13):7004-11.

10. Venere M, Horbinski C, Crish JF, Jin X, Vasanji A, Major J, Burrows AC, Chang C, Prokop J, Wu Q, et al. The mitotic kinesin KIF11 is a driver of invasion, proliferation, and self-renewal in glioblastoma. Science translational medicine. 2015;7(304):304ra143.

11. Tomita Y, Yuno A, Tsukamoto H, Senju S, Kuroda Y, Hirayama M, Irie A, Kawahara K, Yatsuda J, Hamada A, et al. Identification of promiscuous KIF20A long peptides bearing both CD4 + and CD $8+$ T-cell epitopes: KIF20A-specific CD4 + T-cell immunity in patients with malignant tumor. Clinical cancer research: an official journal of the American Association for Cancer Research. 2013;19(16):4508-20.

12. Taniwaki M, Takano A, Ishikawa N, Yasui W, Inai K, Nishimura H, Tsuchiya E, Kohno N, Nakamura Y, Daigo Y. Activation of KIF4A as a prognostic biomarker and therapeutic target for lung cancer. Clinical cancer research: an official journal of the American Association for Cancer Research. 2007;13(22 Pt 1):6624-31.

13. Lucanus AJ, Yip GW. Kinesin superfamily: roles in breast cancer, patient prognosis and therapeutics. Oncogene. 2018;37(7):833-8.

14. Gomez HL, Philco M, Pimentel P, Kiyan M, Monsalvo ML, Conlan MG, Saikali KG, Chen MM, Seroogy $\mathrm{JJ}$, Wolff AA, et al. Phase I dose-escalation and pharmacokinetic study of ispinesib, a kinesin spindle protein inhibitor, administered on days 1 and 15 of a 28-day schedule in patients with no prior treatment for advanced breast cancer. Anti-cancer drugs. 2012;23(3):335-41.

15. Wakui H, Yamamoto N, Kitazono S, Mizugaki H, Nakamichi S, Fujiwara Y, Nokihara H, Yamada Y, Suzuki K, Kanda H, et al. A phase 1 and dose-finding study of LY2523355 (litronesib), an Eg5 inhibitor, in Japanese patients with advanced solid tumors. Cancer Chemother Pharmacol. 2014;74(1):15-23.

16. Infante JR, Patnaik A, Verschraegen CF, Olszanski AJ, Shaheen M, Burris HA, Tolcher AW, Papadopoulos KP, Beeram M, Hynes SM, et al. Two Phase 1 dose-escalation studies exploring 
multiple regimens of litronesib (LY2523355), an Eg5 inhibitor, in patients with advanced cancer. Cancer Chemother Pharmacol. 2017;79(2):315-26.

17. Shah JJ, Kaufman JL, Zonder JA, Cohen AD, Bensinger WI, Hilder BW, Rush SA, Walker DH, Tunquist BJ, Litwiler KS, et al. A Phase 1 and 2 study of Filanesib alone and in combination with low-dose dexamethasone in relapsed/refractory multiple myeloma. Cancer. 2017;123(23):4617-30.

18. Ocio EM, Motlló C, Rodríguez-Otero P, Martínez-López J, Cejalvo MJ, Martín-Sánchez J, Bladé J, García-Malo MD, Dourdil MV, García-Mateo A, et al. Filanesib in combination with pomalidomide and dexamethasone in refractory MM patients: safety and efficacy, and association with alpha 1-acid glycoprotein (AAG) levels. Phase Ib/II Pomdefil clinical trial conducted by the Spanish MM group. $\mathrm{Br}$ J Haematol. 2021;192(3):522-30.

19. Hernández-García S, San-Segundo L, González-Méndez L, Corchete LA, Misiewicz-Krzeminska I, Martín-Sánchez M, López-Iglesias AA, Algarín EM, Mogollón P, Díaz-Tejedor A, et al. The kinesin spindle protein inhibitor filanesib enhances the activity of pomalidomide and dexamethasone in multiple myeloma. Haematologica. 2017;102(12):2113-24.

20. O'Connor OA, Gerecitano J, Van Deventer H, Hainsworth J, Zullo KM, Saikali K, Seroogy J, Wolff A, Escandón R. The addition of granulocyte-colony stimulating factor shifts the dose limiting toxicity and markedly increases the maximum tolerated dose and activity of the kinesin spindle protein inhibitor SB-743921 in patients with relapsed or refractory lymphoma: results of an international, multicenter phase I/II study. Leukemia lymphoma. 2015;56(9):2585-91.

21. Jones R, Vuky J, Elliott T, Mead G, Arranz JA, Chester J, Chowdhury S, Dudek AZ, Müller-Mattheis V, Grimm MO, et al. Phase II study to assess the efficacy, safety and tolerability of the mitotic spindle kinesin inhibitor AZD4877 in patients with recurrent advanced urothelial cancer. Investig New Drugs. 2013;31(4):1001-7.

22. Yoon JR, Whipple RA, Balzer EM, Cho EH, Matrone MA, Peckham M, Martin SS. Local anesthetics inhibit kinesin motility and microtentacle protrusions in human epithelial and breast tumor cells. Breast cancer research treatment. 2011;129(3):691-701.

23. Zhang W, Zhai L, Wang Y, Boohaker RJ, Lu W, Gupta VV, Padmalayam I, Bostwick RJ, White EL, Ross LJ, et al. Discovery of a novel inhibitor of kinesin-like protein KIFC1. Biochem J. 2016;473(8):102735 .

24. Wu J, Mikule K, Wang W, Su N, Petteruti P, Gharahdaghi F, Code E, Zhu X, Jacques K, Lai Z, et al: Discovery and mechanistic study of a small molecule inhibitor for motor protein KIFC1. ACS chemical biology 2013, 8(10):2201-2208.

25. Scanlan MJ, Gout I, Gordon CM, Williamson B, Stockert E, Gure AO, Jäger D, Chen YT, Mackay A, O'Hare MJ, et al. Humoral immunity to human breast cancer: antigen definition and quantitative analysis of mRNA expression. Cancer Immun. 2001;1:4.

26. Wang J, Guo X, Xie C, Jiang J. KIF15 promotes pancreatic cancer proliferation via the MEK-ERK signalling pathway. British journal of cancer. 2017;117(2):245-55. 
27. Hill E, Clarke M, Barr FA. The Rab6-binding kinesin, Rab6-KIFL, is required for cytokinesis. EMBO J. 2000;19(21):5711-9.

28. Taniuchi K, Nakagawa H, Nakamura T, Eguchi H, Ohigashi $H$, Ishikawa O, Katagiri T, Nakamura Y. Down-regulation of RAB6KIFL/KIF20A, a kinesin involved with membrane trafficking of discs large homologue 5, can attenuate growth of pancreatic cancer cell. Cancer research. 2005;65(1):105-12.

29. Kanehira M, Katagiri T, Shimo A, Takata R, Shuin T, Miki T, Fujioka T, Nakamura Y. Oncogenic role of MPHOSPH1, a cancer-testis antigen specific to human bladder cancer. Cancer research. 2007;67(7):3276-85.

30. Liu XR, Cai Y, Cao X, Wei RC, Li HL, Zhou XM, Zhang KJ, Wu S, Qian QJ, Cheng B, et al. A new oncolytic adenoviral vector carrying dual tumour suppressor genes shows potent anti-tumour effect. J Cell Mol Med. 2012;16(6):1298-309.

31. Muhia M, Thies E, Labonté D, Ghiretti AE, Gromova KV, Xompero F, Lappe-Siefke C, HermansBorgmeyer I, Kuhl D, Schweizer M, et al. The Kinesin KIF21B Regulates Microtubule Dynamics and Is Essential for Neuronal Morphology, Synapse Function, and Learning and Memory. Cell reports. 2016;15(5):968-77.

32. Ghiretti AE, Thies E, Tokito MK, Lin T, Ostap EM, Kneussel M, Holzbaur ELF. Activity-Dependent Regulation of Distinct Transport and Cytoskeletal Remodeling Functions of the Dendritic Kinesin KIF21B. Neuron. 2016;92(4):857-72.

33. van Riel WE, Rai A, Bianchi S, Katrukha EA, Liu Q, Heck AJ, Hoogenraad CC, Steinmetz MO, Kapitein LC, Akhmanova A: Kinesin-4 KIF21B is a potent microtubule pausing factor. eL ife 2017, 6.

34. Walczak CE, Gayek S, Ohi R. Microtubule-depolymerizing kinesins. Annu Rev Cell Dev Biol. 2013;29:417-41.

35. He M, Subramanian R, Bangs F, Omelchenko T, Liem KF Jr, Kapoor TM, Anderson KV. The kinesin-4 protein Kif7 regulates mammalian Hedgehog signalling by organizing the cilium tip compartment. Nat Cell Biol. 2014;16(7):663-72.

36. Zhu C, Bossy-Wetzel E, Jiang W. Recruitment of MKLP1 to the spindle midzone/midbody by INCENP is essential for midbody formation and completion of cytokinesis in human cells. Biochem $\mathrm{J}$. 2005;389(Pt 2):373-81.

37. Gao CT, Ren J, Yu J, Li SN, Guo XF, Zhou YZ. KIF23 enhances cell proliferation in pancreatic ductal adenocarcinoma and is a potent therapeutic target. Annals of translational medicine. 2020;8(21):1394.

38. Goldman MJ, Craft B, Hastie M, Repečka K, McDade F, Kamath A, Banerjee A, Luo Y, Rogers D, Brooks $\mathrm{AN}$, et al. Visualizing and interpreting cancer genomics data via the Xena platform. Nature biotechnology. 2020;38(6):675-8.

39. Tang Z, Li C, Kang B, Gao G, Li C, Zhang Z. GEPIA: a web server for cancer and normal gene expression profiling and interactive analyses. Nucleic acids research. 2017;45(W1):W98-w102.

40. Rhodes DR, Kalyana-Sundaram S, Mahavisno V, Varambally R, Yu J, Briggs BB, Barrette TR, Anstet MJ, Kincead-Beal C, Kulkarni P, et al. Oncomine 3.0: genes, pathways, and networks in a collection of 
18,000 cancer gene expression profiles. Neoplasia (New York NY). 2007;9(2):166-80.

41. Uhlen M, Zhang C, Lee S, Sjöstedt E, Fagerberg L, Bidkhori G, Benfeitas R, Arif M, Liu Z, Edfors F, et al: A pathology atlas of the human cancer transcriptome. Science (New York, NY) 2017, 357(6352).

42. Győrffy B, Surowiak P, Budczies J, Lánczky A. Online survival analysis software to assess the prognostic value of biomarkers using transcriptomic data in non-small-cell lung cancer. PloS one. 2013;8(12):e82241.

43. Nagy Á, Lánczky A, Menyhárt O, Győrffy B. Validation of miRNA prognostic power in hepatocellular carcinoma using expression data of independent datasets. Scientific reports. 2018;8(1):9227.

44. Li C, Pak D, Todem D. Adaptive lasso for the Cox regression with interval censored and possibly left truncated data. Stat Methods Med Res. 2020;29(4):1243-55.

45. Engebretsen S, Bohlin J. Statistical predictions with glmnet. Clinical epigenetics. 2019;11(1):123.

46. Zheng Y, Heagerty PJ. Prospective accuracy for longitudinal markers. Biometrics. 2007;63(2):33241.

47. Iasonos A, Schrag D, Raj GV, Panageas KS. How to build and interpret a nomogram for cancer prognosis. Journal of clinical oncology: official journal of the American Society of Clinical Oncology. 2008;26(8):1364-70.

48. Gerds TA, Andersen PK, Kattan MW. Calibration plots for risk prediction models in the presence of competing risks. Statistics in medicine. 2014;33(18):3191-203.

49. Gao J, Aksoy BA, Dogrusoz U, Dresdner G, Gross B, Sumer SO, Sun Y, Jacobsen A, Sinha R, Larsson E, et al. Integrative analysis of complex cancer genomics and clinical profiles using the cBioPortal. Sci Signal. 2013;6(269):pl1.

50. Szklarczyk D, Gable AL, Lyon D, Junge A, Wyder S, Huerta-Cepas J, Simonovic M, Doncheva NT, Morris JH, Bork P, et al. STRING v11: protein-protein association networks with increased coverage, supporting functional discovery in genome-wide experimental datasets. Nucleic acids research. 2019;47(D1):D607-d613.

51. Yu G, Wang LG, Han Y, He QY. clusterProfiler: an R package for comparing biological themes among gene clusters. Omics: a journal of integrative biology. 2012;16(5):284-7.

52. Walter W, Sánchez-Cabo F, Ricote M. GOplot: an R package for visually combining expression data with functional analysis. Bioinformatics. 2015;31(17):2912-4.

53. Li T, Fan J, Wang B, Traugh N, Chen Q, Liu JS, Li B, Liu XS. TIMER: A Web Server for Comprehensive Analysis of Tumor-Infiltrating Immune Cells. Cancer research. 2017;77(21):e108-10.

\section{Figures}


PAAD

Nomal

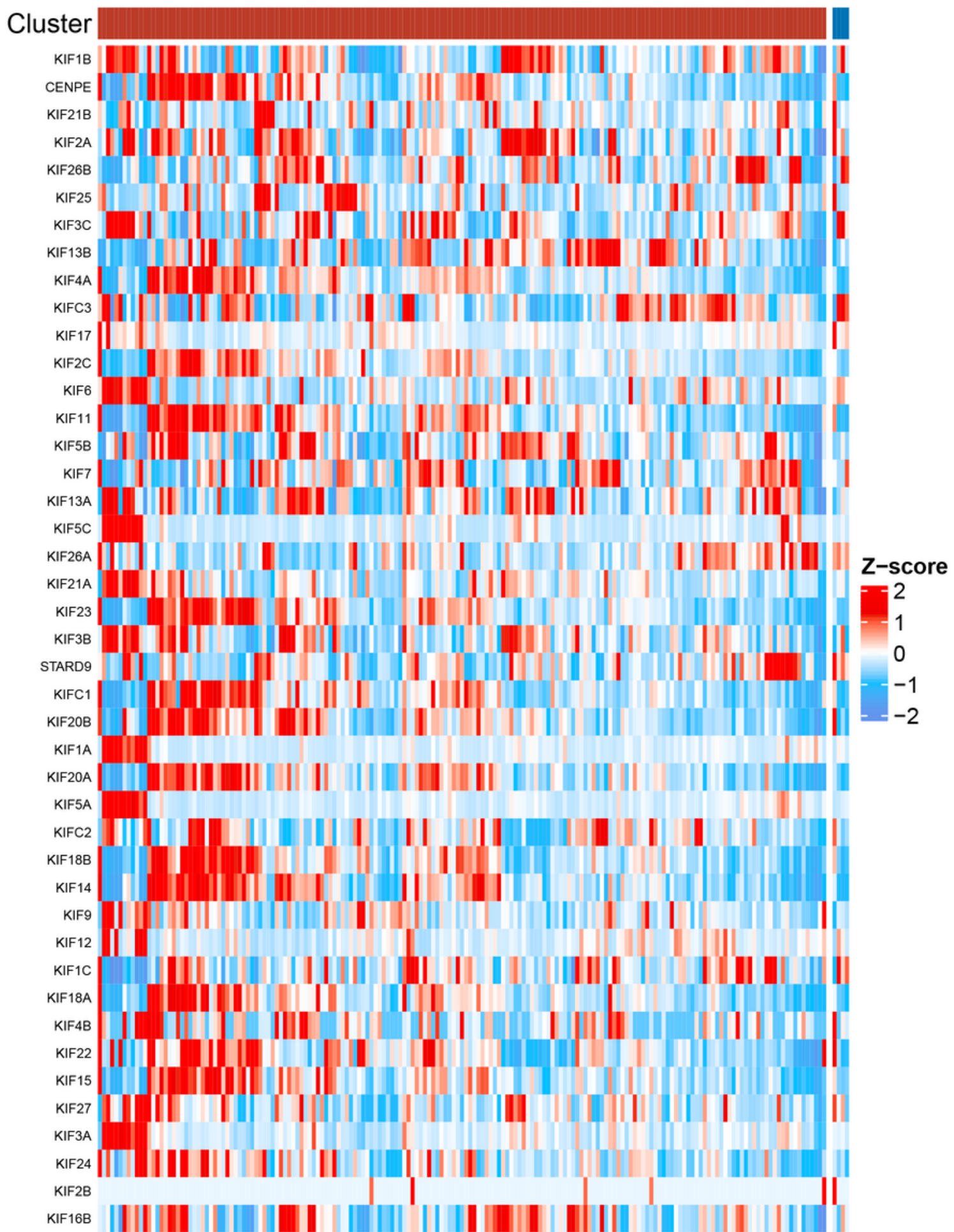

Figure 1

Expression profiles of kinesin superfamily in Pancreatic Adenocarcinoma. 


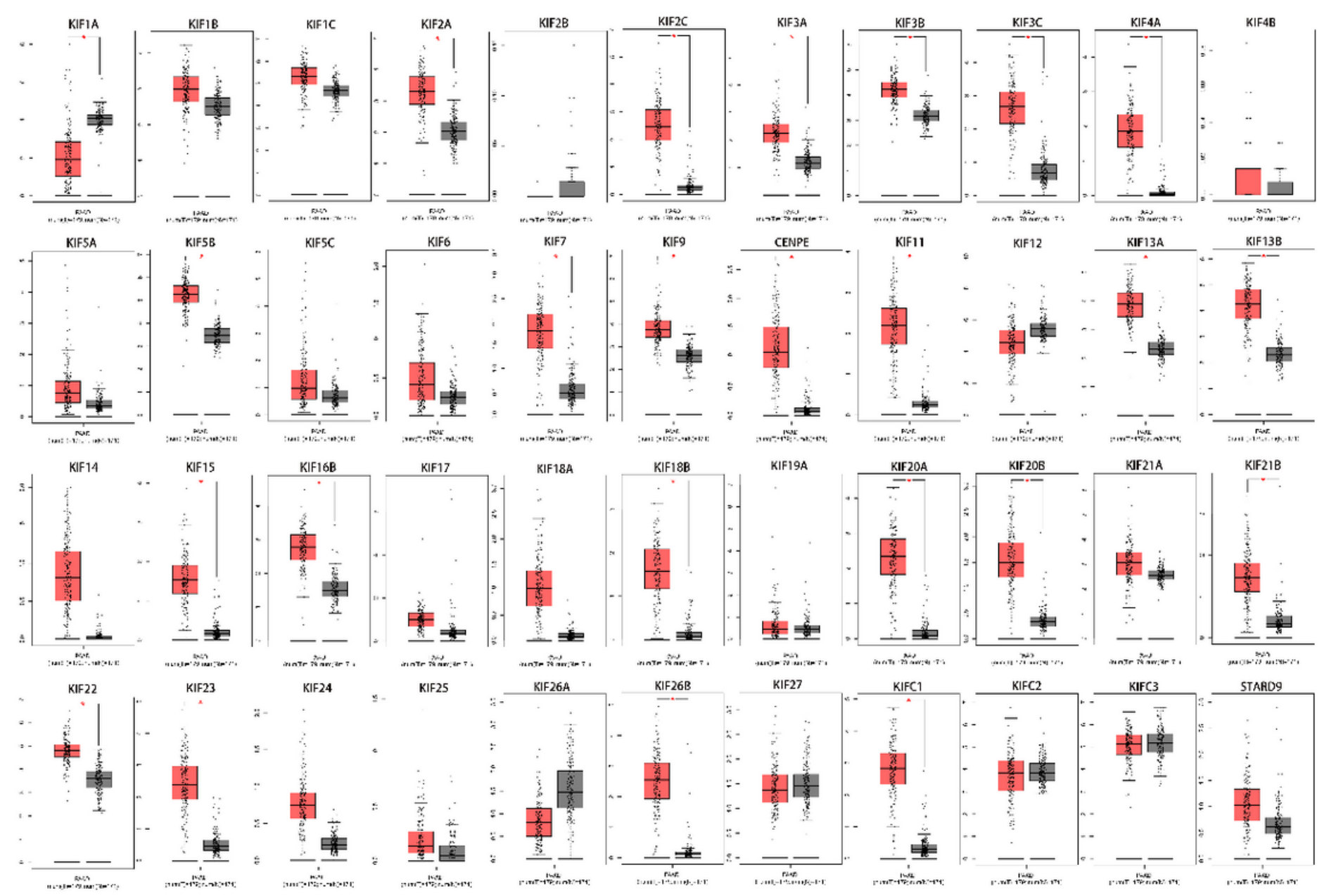

Figure 2

Expression boxplot of each kinesin superfamily members (KIF1A, KIF1B, KIF1C, KIF2A, KIF2B, KIF2C, KIF3A, KIF3B, KIF3C, KIF4A, KIF4B, KIF5A, KIF5B, KIF5C, KIF6, KIF7, KIF11, KIF9, KIF10, CENPE, KIF12, KIF13A, KIF13B, KIF14, KIF15, STARD9, KIF16B, KIF17, KIF18A, KIF18B, KIF19A, KIF20A, KIF20B, KIF21A, KIF21B, KIF22, KIF23, KIF24, KIF25, KIF26A, KIF26B, KIF27, KIFC1, KIFC2, KIFC3) using both TCGA-PAAD and matched GTEx-normal data (Tumor $=179 ;$ Normal $=171$ ); $* \mathrm{P}<0.05 ; * \star \mathrm{P}<0.01 ; * \star \star \mathrm{P}<0.001$ 

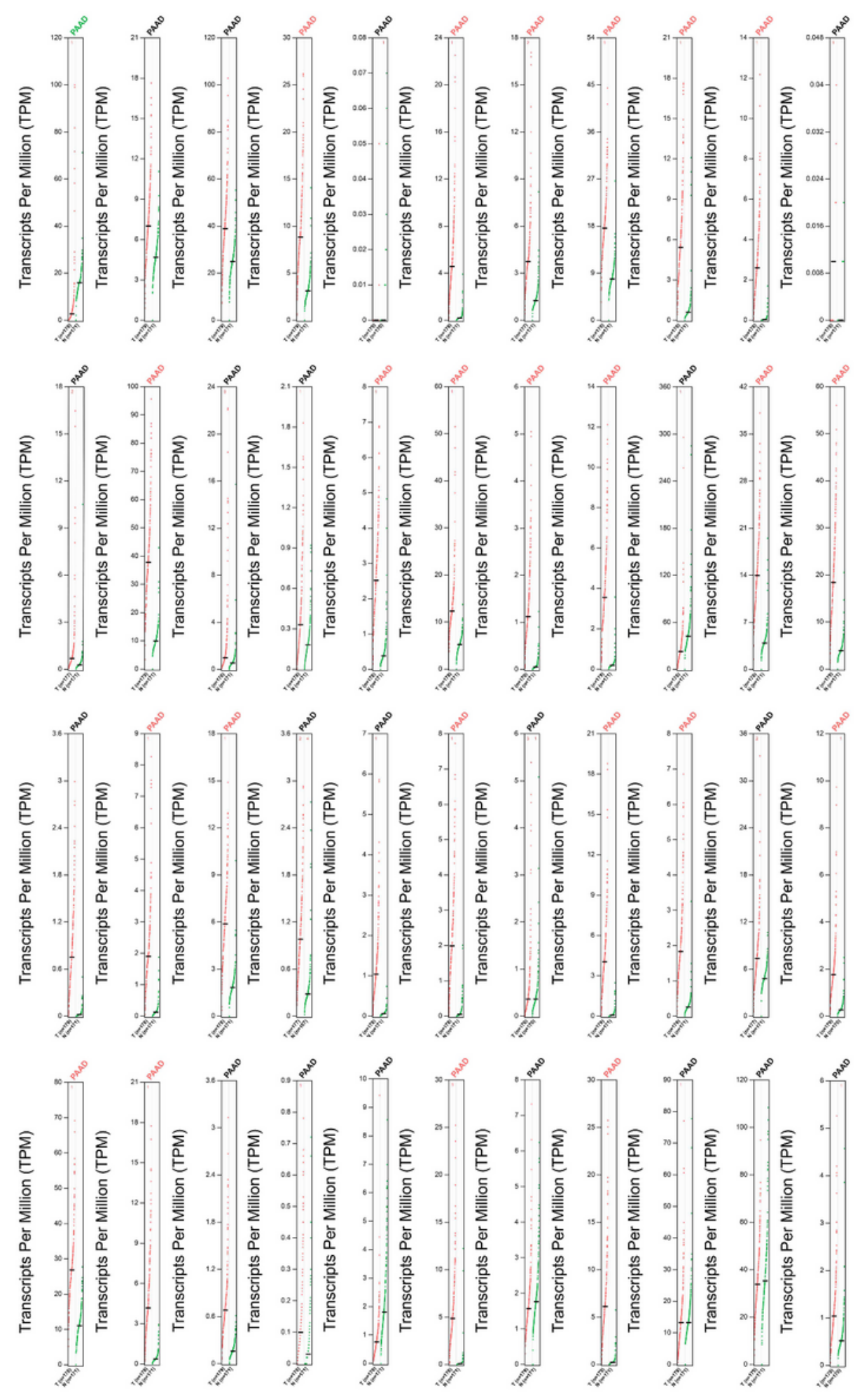

\section{Figure 3}

Expression profile of each kinesin superfamily members (KIF1A, KIF1B, KIF1C, KIF2A, KIF2B, KIF2C, KIF3A, KIF3B, KIF3C, KIF4A, KIF4B, KIF5A, KIF5B, KIF5C, KIF6, KIF7, KIF11, KIF9, KIF10, CENPE, KIF12, KIF13A, KIF13B, KIF14, KIF15, STARD9, KIF16B, KIF17, KIF18A, KIF18B, KIF19A, KIF20A, KIF20B, KIF21A, KIF21B, KIF22, KIF23, KIF24, KIF25, KIF26A, KIF26B, KIF27, KIFC1, KIFC2, KIFC3) using both TCGA-PAAD and matched GTEx-normal data $($ Tumor $=179 ;$ Normal $=171)$ 


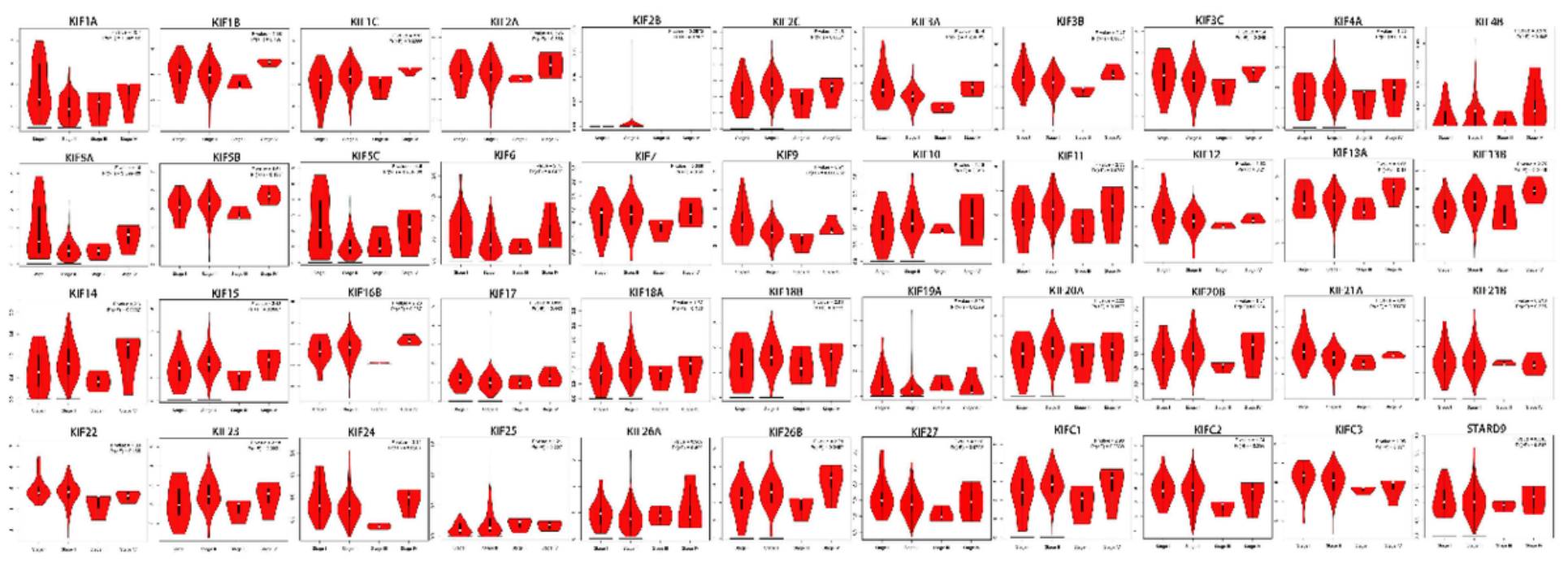

Figure 4

Correlation between KIFs Expression and Tumor Stage in PAAD Patients (GEPIA)

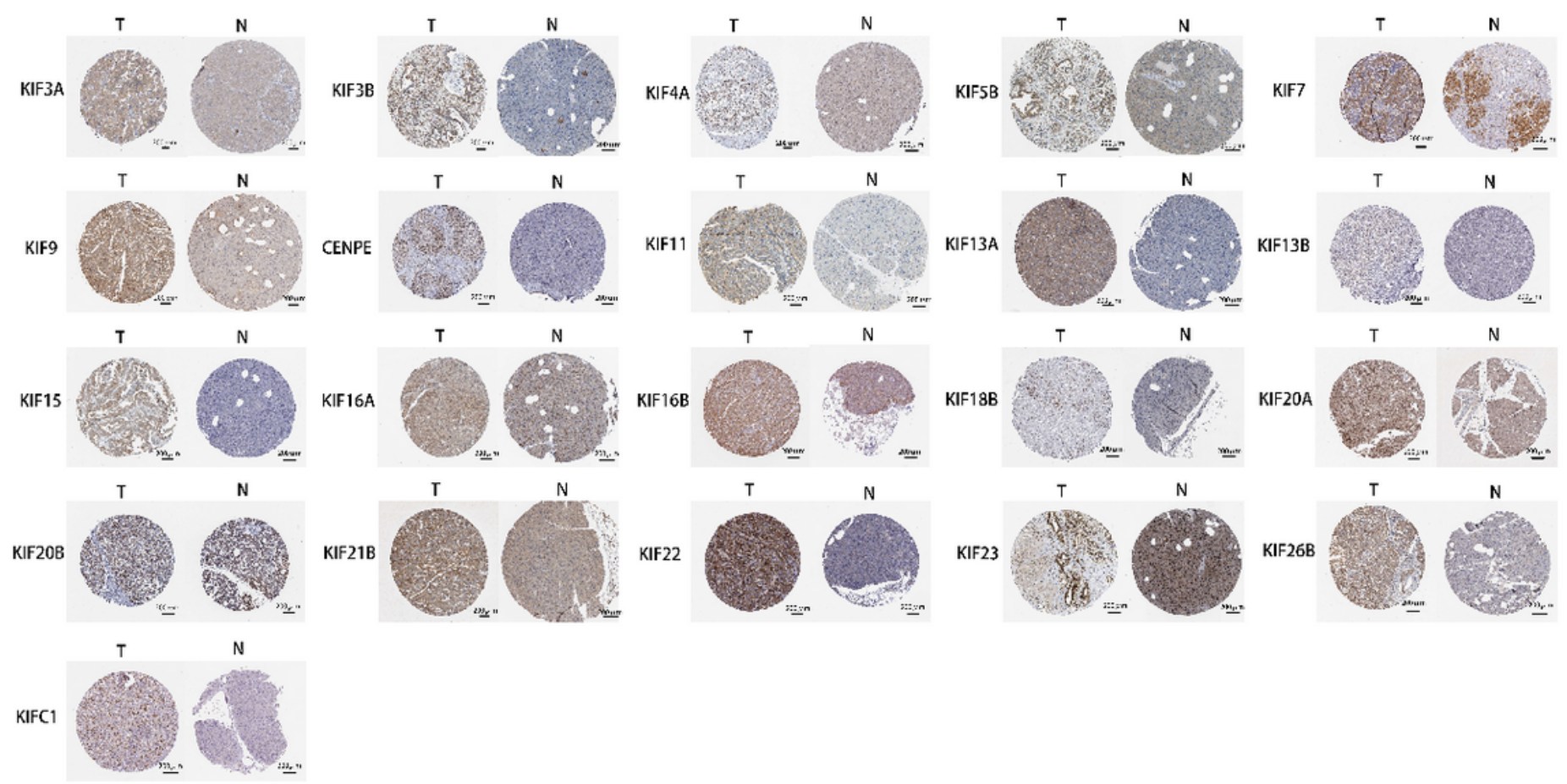

Figure 5

The Expression of KIFs in PAAD (HPA) 

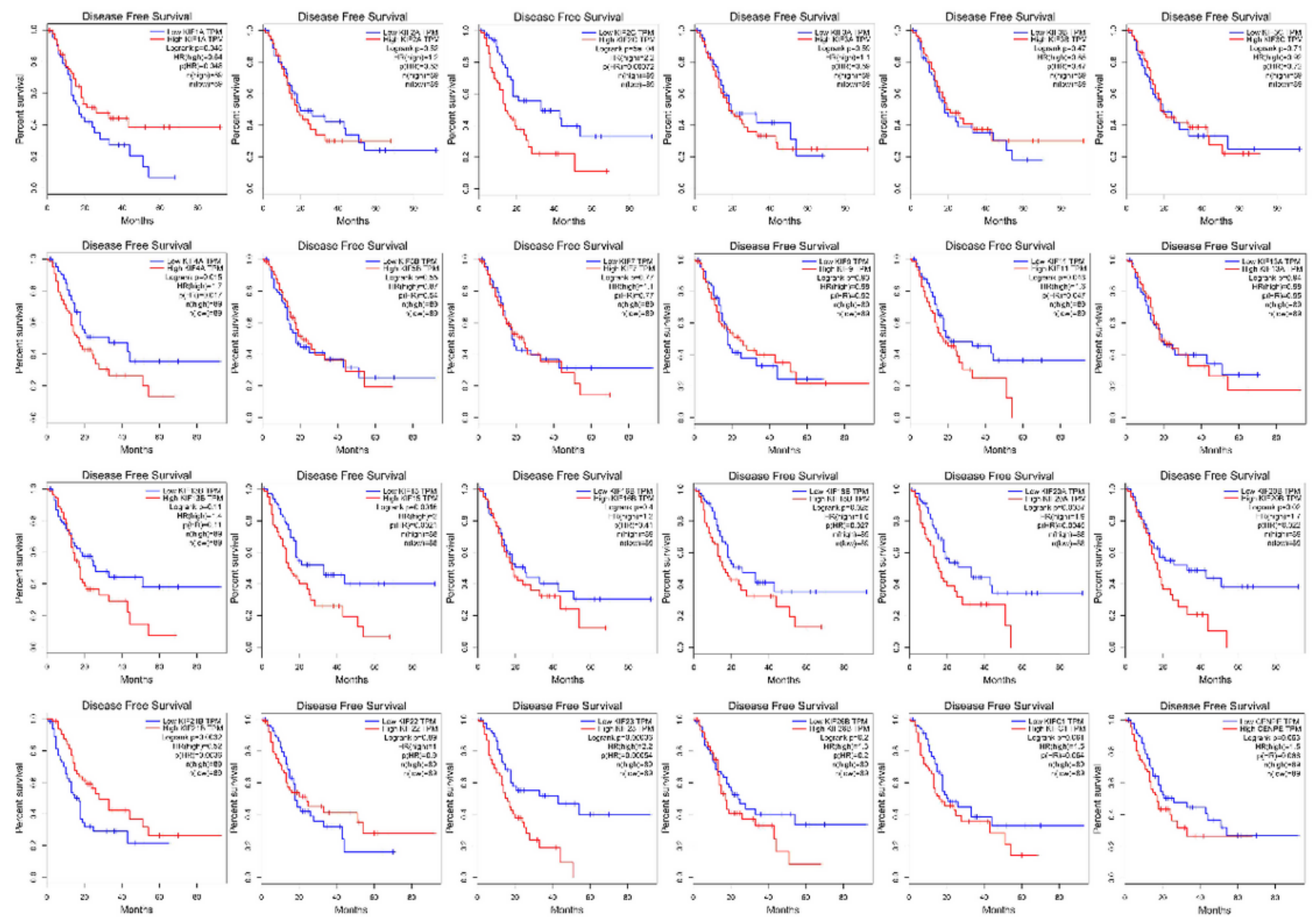

Figure 6

The prognostic value of different expressed KIFs in PAAD patients in the RFS curve (Kaplan-Meier plotter). 

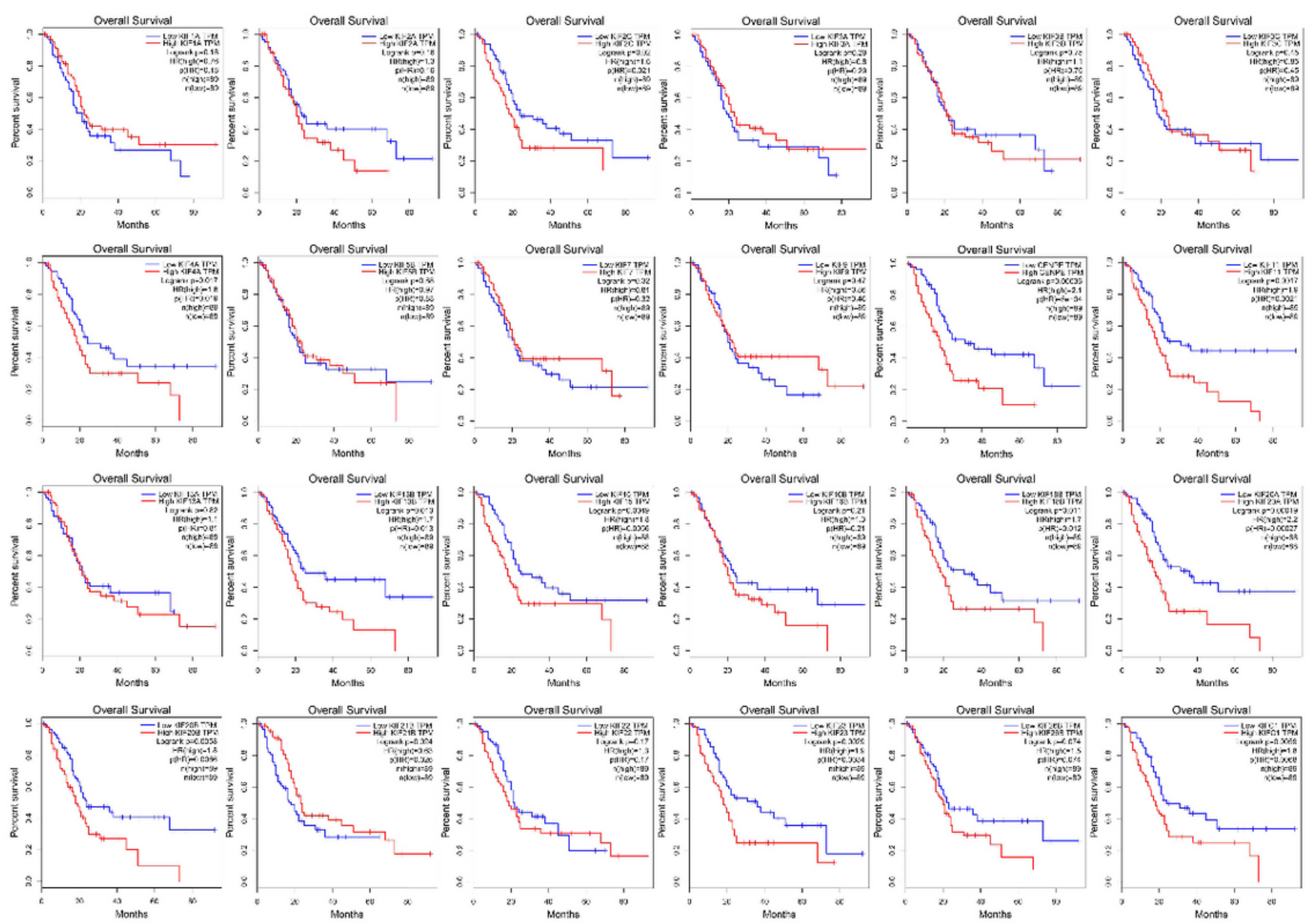

Figure 7

The prognostic value of different expressed KIFs in PAAD patients in the OS curve (Kaplan-Meier plotter). 

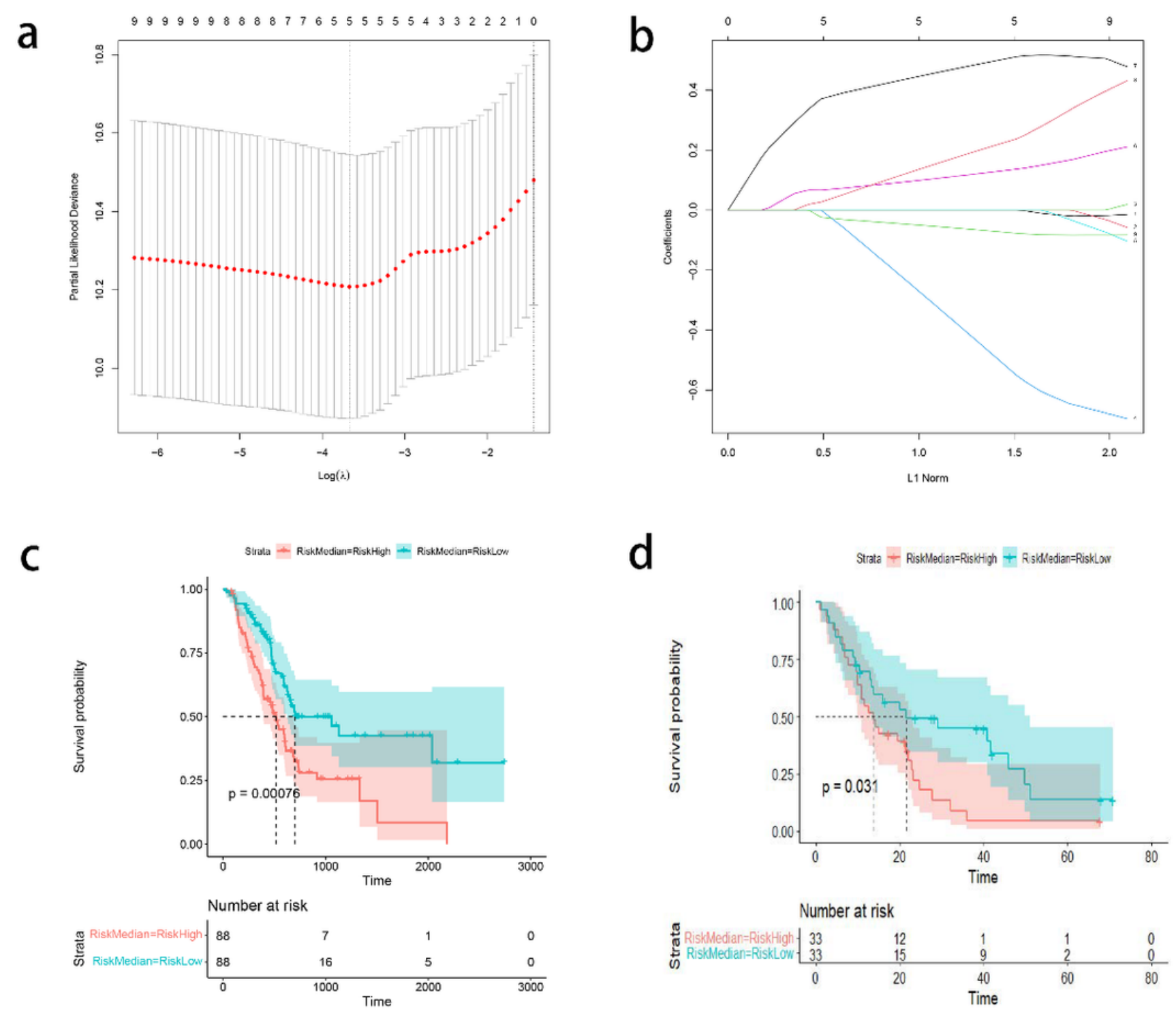

\section{Figure 8}

LASSO regression of overall survival related KIFs. a Cvfit plot for the selection of LASSO regression lambda value as best cutoff. b LASSO regression of overall survival related KIFs (KIF2C, KIF4A, KIF11, KIF15, KIF18B, KIF20A, KIF20B, KIF23, KIF21B).c Validation of prognostic value of the 5-KIFs-based risk score generated by LASSO regression using survival analyses with TCGA-PAAD data. $d$ Validation of prognostic value of the 5-KIFs-based risk score generated by LASSO regression using survival analyses with GEO dataset (GSE62452). 
A

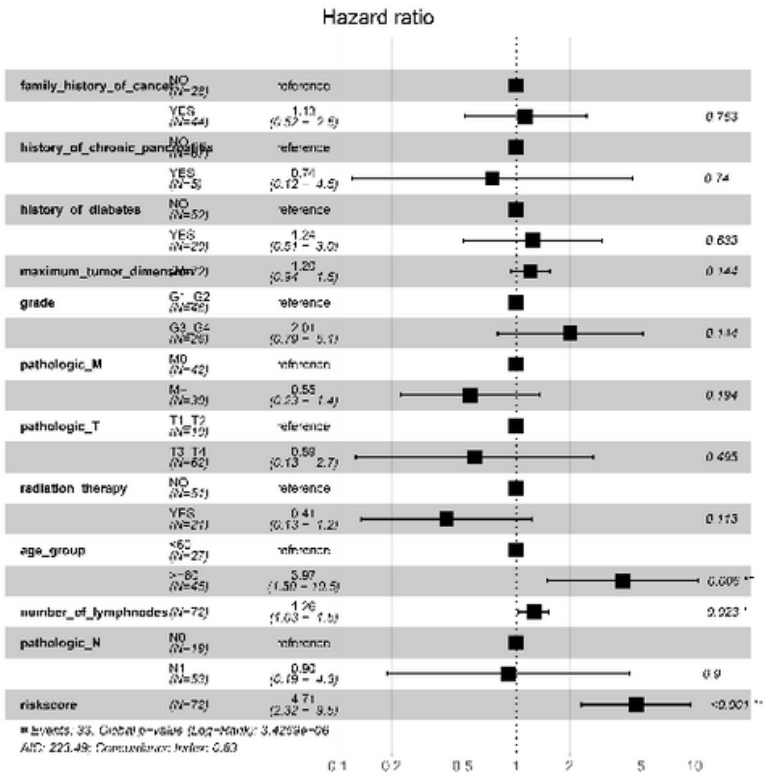

c

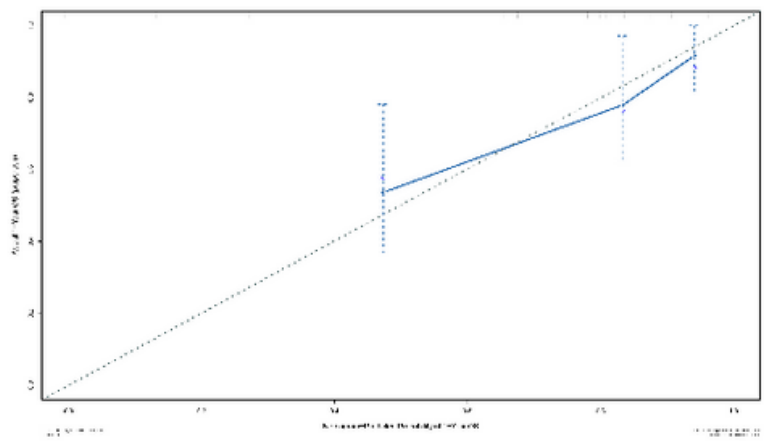

B

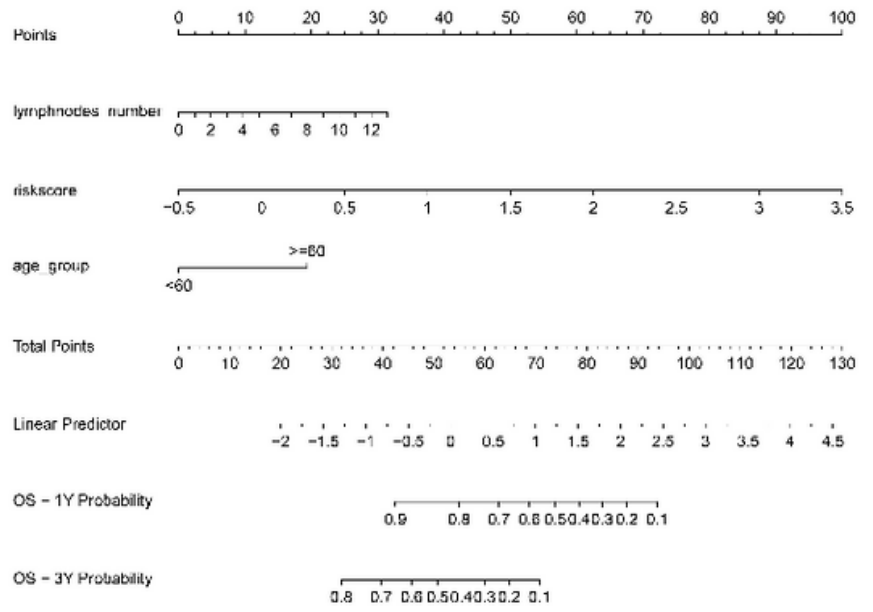

D

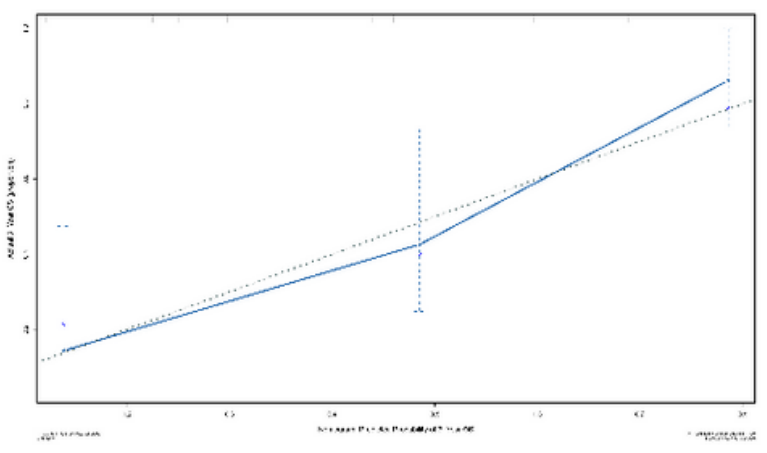

Figure 9

A. Multivariate survival analyses of clinical and pathologic information of PAAD patients. B. Nomogram constructed with pathologic information, clinical information and 5-KIFs-based risk score for prediction of 1/3-year OS of PAAD patients. C. Calibration plot done to self-validate the 1-year survival prediction efficacy of nomogram constructed. D. Calibration plot done to self-validate the 3-year survival prediction efficacy of nomogram constructed 
A

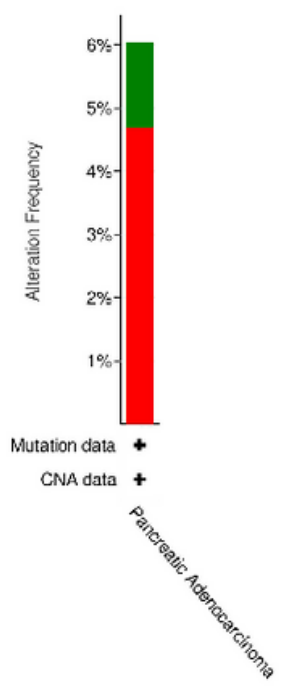

- Mutation - Amplification

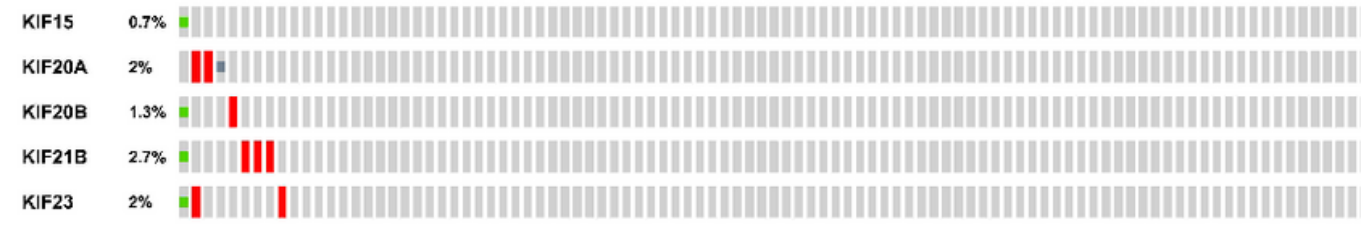

Genetic Alteration

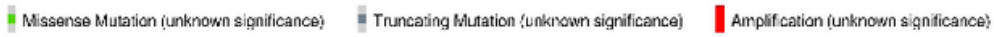

|l No altarations

\section{Figure 10}

Summary of alterations in the 5-KIFs expressed in $\operatorname{PAAD}(\mathrm{A})$. OncoPrint visual summary of alteration on a query of the 5 -KIFs family members(B).
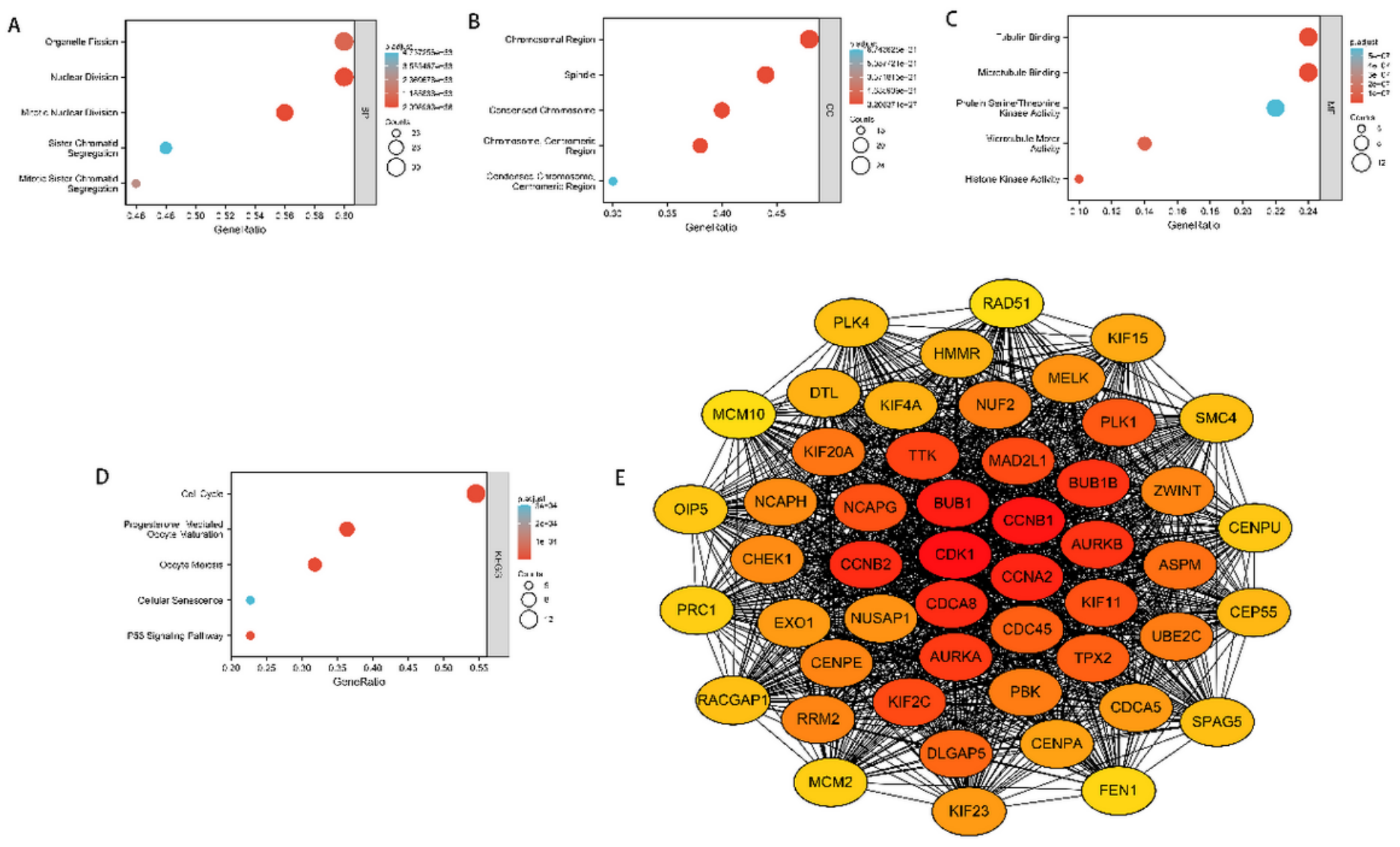

Figure 11 
The functions of the 5-KIFs and genes significantly associated with KIFs alterations were predicted by the analysis of gene ontology (GO) by DAVID tools. GO enrichment analysis predicted the functional roles of target host genes based on three aspects, including (A) biological processes, (B) cellular components, and (C) molecular functions. The functions of KIFs and genes significantly associated with KIF alterations were predicted by the analysis of Kyoto Encyclopedia of Genes and Genomes (KEGG) by DAVID tools(D). The network for KIFs and the 50 most frequently altered neighbor genes(E).
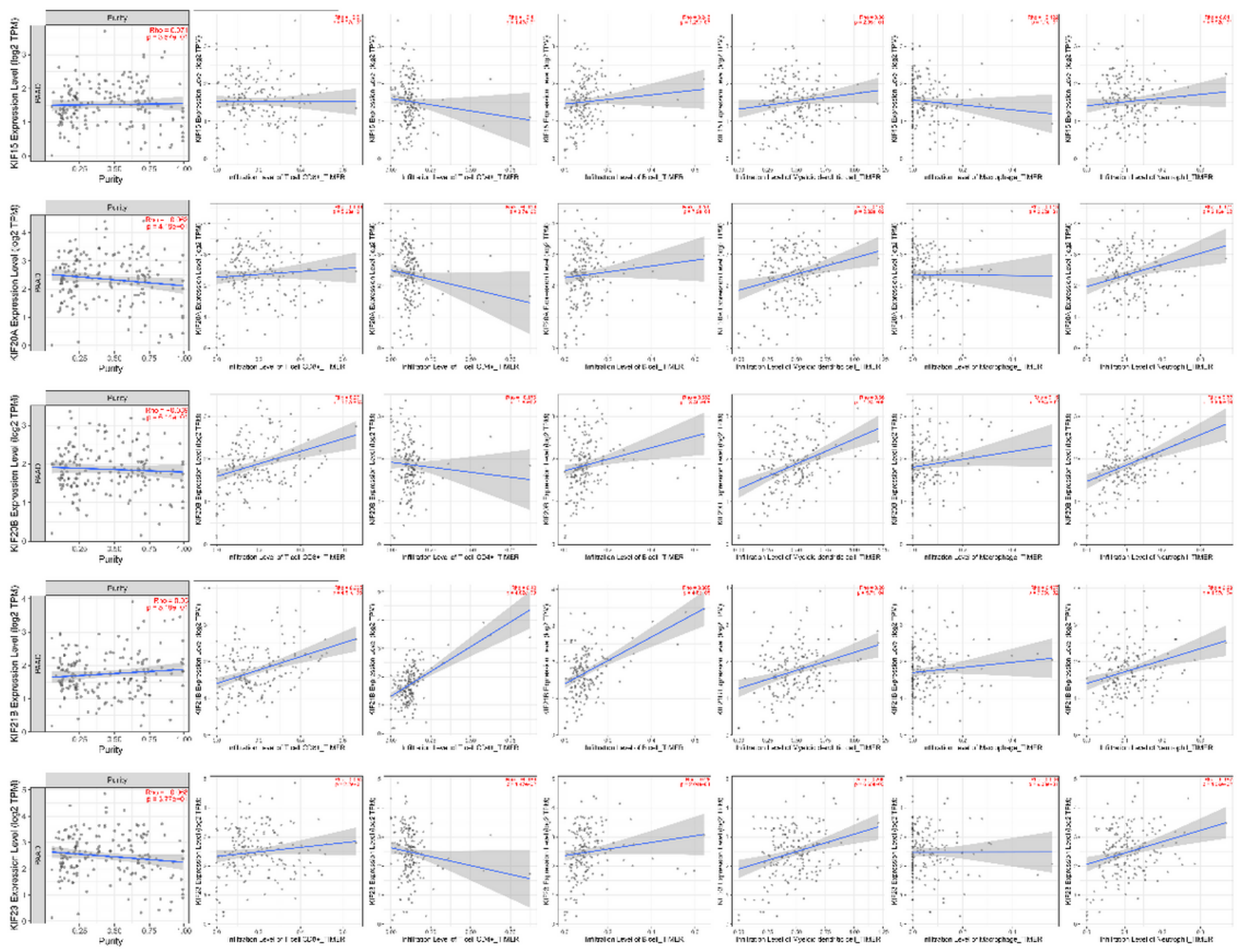

\section{Figure 12}

The Relationship between the 5-KIFs (KIF15, KIF20A, KIF20B, KIF21BA, KIF23) Expression Levels and Immune Infiltration Levels in PAAD (TIMER). The correlation between the abundance of immune cell and the expression of KIFs. 